\title{
The Preparation of Gas for Balloons
}

\author{
What Chemistry Has Done for Ballooning
}

\section{By A. Sander}

TECHNICAL chemistry has contributed a very im. portant share to the successful development of aerial navigation within the past few years; for a number of products of chemical industry function as important auxiliaries in the construction and operation, both of dirigible balloons and of aeroplanes. It is only necessary to call to mind the several special steels and the light aluminium magnesium alloys, which are of the highest importance as materials of construction for aeronautical purposes; or the Indin rubber used for impregnating the light and yet highly resistant and tough gas-proof balloon fabric; there is also the very important item of gasoline and oil, on the proper chemical purification of which the safe operation of the motor depends in a large measure; lastly there is the important problem of the production of the balloon gas, which places before the technical chemist a number of difficult problems for solution. Just as in a flying machine the motor is the vital point, so in ballooning the preparation of the buoyant gas is of fundamental importance.

Before turning to the consideration of the technical production of the balloon gas, it will be well first of all to briefly call to mind its purpose and the requirements which it is called upon to fulfill. The purpose of balloon gas is, of course, to lift a car, which togethe with its passengers, ballast, and apparatus, has a considerable weight. The dimensions of the balloon and car must be so chosen that the weight of the entire system is less than that of the air displaced thereby. Hence the first demand laid upon balloon gas is that it shall be very light as compared with air. This, however, is not by any means the only condition which has to be satisfied. The ideal balloon gas, which un fortunately is as yet but an ideal, should in addition to low density possess the following properties: It must not attack the balloon envelope and should be incombustible, in other words it should be chemically inert. Furthermore, it should be as little as possible affected by changes in temperature, it should be readily liquefiable, non-poisonous, and last but not least, it must be cheap. While a large number of gases might be cited which fulfill more or less satisfactorily the first condition, that of low density, when we strike from such a list all those gases which fail to satisfy one or other of the remaining conditions, we are left with practically only three substances as having hitherto been used for a balloon filling, and they are heated air, illuminating gas, and hydrogen. We will briefly consider each of these in turn:

I. Heated Air.-The art of aerial navigation may be said to date from the year 1783 , in which the Montgolfier Brothers made a public ascent in their home in Brittany, by the aid of a paper balloon covered with linen and inflated with hot air. It is on record that these same inventors had in the first instance experimented with steam as a filling for the balloon, the idea being suggested to their mind by the sight of the clouds drifting in the sky. In point of fact steam would make a very fair gas for ballooning were it not for the impossibility of preventing its condensation. The Montgolfier hot-air balloon had a number of obvious limita tions. The fact that fire had to be carried on boar placed the occupants in considerable jeopardy owing to the risk of the balloon itself being set in flames. Furthermore, the extreme height to which it could ascend was only about 8,000 feet, for in order to rise higher than this it would be necessary to heat the air above $100 \mathrm{deg}$. C., which could not be done without injuring the balloon cover.

II. Illuminating Gas.-Of much greater importance than hot air for ballooning purposes is the use of illuminating gas. Its introduction into the art is usually ascribed to an Englishman, Greene, in 1818; it has, however, been demonstrated from old documents by Dutch chemists that illuminating gas was used a early as 1783 in their country, that is to say, in the very same year in which the brothers Montgolfier made their first ascent.

At the present day gas works are the principal centers from which most balloon ascents take place, and the quantity of gas consumed for this purpose is rapidly increasing. A very brief resume of the preparation of illuminating gas may here suffice, inasmuch as its mod of manufacture is more or less a matter of common knowledge owing to its extended use in everyday life. As is well known, illuminating gas is prepared by th "dry distillation" of coal. This process is usually carried on in fire clay retorts arranged in batteries of six to twelve in one furnace. The gas issuing from

\footnotetext{
*Adapted from Zeitschrift für angewandte Chemic.
}

retorts is passed through an elaborate and extended series of apparatus in which it is cooled and freed from certain impurities. The purified gas is collected in so-called gasometers, whence it is forced into the mains for general distribution. Illuminating gas is colorless, but readily recognized by its odor. It is not a chemical individual, but a mixture of gases, its principal constituent, amounting to about fifty per cent being hydrogen. The composition of the gas is some what variable, and in consequence its density also fluc tuates between about 0.36 and 0.52 (expressed in the scale which gives to air the density 1). This cor responds to a lifting power of 0.052 to 0.39 pound per cubic foot. As regards density the requirements of balloonists and of the general consumer of illuminat ing gas are at variance, for as a balloon filling a light gas rich in hydrogen is the desideratum, while for illuminating purposes a gas rich in the heavier illu minants is more advantageous. Nevertheless there ar few gas works in Germany whose product has density greater than 0.45 . Heavier gas occurs only wher exclusively silesian coal is distilled, or where much water gas is added.

In the course of the distillation of coal the composition of the gas issuing from the retorts varies considerably, and especially toward the end of the distilla tion a very light gas rich in hydrogen is obtained. It has therefore been suggested to collect the last portions, which are of comparatively low illuminatin value, separately, and to utilize them for ballooning purposes. This mode of procedure would, however, introduce considerable complication into the manu facture of coal gas, and has therefore found no application in practice. On the other hand a method which of recent years has been applied with much success, consists in converting the purified gas into a lighte product by heating it at a high temperature. Thi process is not altogether a new invention; in the yea 1894 Bunte and others had observed that by this treat ment ordinary illuminating gas can be made to yield a product containing about 80 per cent hydrogen an having a density of about 0.2 . The process was, how ever, not put into practice until very recently, whe Dr. von Oechelhäuser gave publicity to the fact that a special balloon gas by simply heating illuminating gas to 1,200 deg. C. The apparatus used is very sim ple. The gas is passed through highly heated retort charged with small coke. At the temperature indicated the hydro-carbons are split up into their constituents, as for example:

\section{$\mathrm{CH}_{4}=\mathrm{C}+2 \mathrm{H}_{2}$}

This change is accompanied by an increase in volume of 20 per cent, while the carbon is deposited upon the retort walls in the form of soot or graphite. The gas produced is passed through a small purifying plant and into a gas holder. The additional cost of converting the ordinary illuminating gas into balloon gas is just about the same as the cost of distributing the ordinary gas to consumers, so that the price of balloon gas at the works should be about the same as the price of illuminating gas at the point of consumption. The specific gravity of the balloon gas fluctuates between 0.225 and 0.3 , with an average of about 0.27 and a lifting power of about 59 pounds per cubic foot. This corresponds to $\$ 26$ to $\$ 34$ per ton of lifting power (German conditions). The gas contains about 80 per cent hydrogen and only 5 per cent of methane; it is free from benzine and other hydrocarbon which would attack the balloon envelope; it has a very much weaker odor than ordinary coal gas, and is much less sensitive to temperature changes.

While this process of making balloon gas represents a very considerable advance for the purposes of ordinary balloons, it is hardly likely that it will ever have any value for dirigible balloons. For on account of the great weight of such crafts it is necessary to employ the highest buoyancy available, so that the only gas which enters into consideration is hydrogen.

III. Hydrogen.-The lightest of all known gases is hydrogen, whose density compared with air is 0.07 . It has the further advantage for ballooning purpose of being comparatively non-sensitive toward temperature changes. It is very hard to liquefy, hardly soluble in water, and diffuses with great ease even through the smallest openings. This last is the reason why the attempts of the brothers Montgolfier to fill paper balloon envelopes with hydrogen were entirely unsuccessful. The physicist, Charles of Paris, was the first to succeed in sending up a balloon filled with hydrogen.
He prepared the gas by the action of dilute acid upon iron. The balloon envelope was made of silk taffeta. Although its volume was less than 1,400 cubic feet, it took three days and three nights to fill, and required no less than 1,100 pounds of iron filings and 550 pounds of acid. The method which Charles used for generat ing the gas was extremely wasteful of material. Coutelle's attempt to prepare hydrogen on a large scale by passing steam over red hot iron did not prove well adapted for large scale working. A great step forward was made by Renard and Giffard, who used the same reaction as Charles, but modified the process by feeding continually fresh quantities of acid to the iron while drawing off the iron sulphate liquor. In this way a much more complete utilization of the iron was secured. Nevertheless this process also represented an extremely costly method of preparing hydrogen. It was only toward the end of the nineties that a new source of hydrogen became available, and almost completely drove out the old process. This new source is the electrolytic hydrogen produced by the Fabrak Griesheim-Elektron, who produced large quantities of hydrogen as a by-product in their electrolytic process of manufacturing caustic alkali. This industry has at the present day reached such a scale that the firm named above produces in its three works daily no less than 700,000 cubic feet of hydrogen. Although of late years the consumption of this gas has increased very greatly, as a matter of fact only a small portion of the gas is at present collected and compressed into steel cylinders at 120 to 150 atmospheres, while the bulk of it is discharged into the atmosphere. The placing upon the market of hydrogen from this source has done much to further the interests and development of aerial navigation in Germany. The electrolytic hydrogen possesses some very remarkable advantages owing to its purity and more especially its freedom from arsenic. This has led to the establishment of plants built especially for the electrolytic production of hydrogen on its own account, that is to say quite apart from the manufacture of alkali. For this purpose the electrolyte is simply water rendered conducting by the addition of a small percentage of acid, or preferably alkali. The advantage of alkali is that its use permits the employment of iron vessels and diminishes the wear and tear of the electrodes.

While electrolytic hydrogen possess the advantages which have been referred to, it unfortunately presents at the same time rather severe drawbacks. Thus the number of costly steel cylinders required for a large production is quite excessive. Furthermore, there is added to the cost of production, which it is true is moderate, the cost of compressing the gas, and, there is obtained as a by-product with every two volumes of hydrogen one volume of oxygen, an outlet for which must be found. Lastly, the item of freight is very considerable, especially in view of the fact that the return of the empty cylinders represents practically the same expenditure as the first shipment of the charged cylinders. In view of the immense gas consumption of the modern air ship, such as for instance the Zeppelin type of fifteen thousand cubic meters (528,000 cubic feet) capacity, it has become imperaive to look for a process of manufacture of hydrogen which would render it possible to produce large quan tities of gas at moderate cost, and which would obviate the use of compressed gas and of steel cylinders. A process of this kind has been installed at Friedrichshafen by the Carbonium Company, based upon the splitting up of acetylene and other hydrocarbons into their components. The gases are decomposed by elec tric sparks into carbon and hydrogen in cylinders provided with stirring gear. The carbon which is deposited in the form of soot is the principal product, and it is claimed that the material so obtained is superior to that from other sources for the production of printers' ink, paint, and other uses. No data are available at the present time as to the practical operation of this process, owing to the fact that a part of the works was destroyed by an explosion last July. Indications, however, seem to be that the process will represent a very economical source of hydrogen.

Another process, which has been worked out by the International Hydrogen Company, of Frankfurt-on-theMain, is based upon the reaction between steam and red hot iron discovered by Lavoisier. As has been mentioned above, an attempt to turn this to technica account had been made years ago by Coutelle, without success. The difficulty was to reduce the oxide of iron formed in the process back into iron, so as to restore 
the raw material and render it available for further use. It has been discovered that pyrites cinders are very well adapte for the process. They are very porous and for this reason readily reducible to metallic iron by passing generator gas over them. On leading steam over the reduced iron so obtained, a very pure 98 per cent hydrogen is readily prepared.

Another very promising process has been introduced by Siemens and Halske, and starts from calcium carbide as raw material. As is well known to every layman at the present day, this substance when treated with water under ordinary conditions yields acetylene. The inventors have discovered that the reaction proceeds along an entirely different course if the calcium carbide is treated with steam at red-heat. The formation of acetylene and other hydrocarbons is almost com pletely avoided and the reaction proceeds essentially according to the following equation

$$
\mathrm{CaC}_{2}+5 \mathrm{H}_{2} \mathrm{O}=\mathrm{CaO}+2 \mathrm{CO}_{2}+5 \mathrm{H}_{2} \text {. }
$$

From the mixtures so obtained the carbon dioxide can be readily extracted by passing the gases over some of the lime, produced in a previous run. The apparatus required is extremely simple and the raw material is cheap, so that this process ought to find wide application.

We now come to a group of processes all starting from the same raw material, namely, water gas. This gas, as is well known, consists of a mixture of about 50 per cent hydrogen and 50 per cent carbon monoxide ( $\mathrm{C} \mathrm{O}$ ). In practice the gas is contaminated with a certain proportion of carbon dioxide and nitrogen. The density of water gas is 0.52 to 0.54 , so that it might be directly utilized for balloon filling; owing to its highly poisonous character, however, this would be very undesirable and dangerous.

The first efforts to use water gas as a source of pure hydrogen dates many years back. At first the endeavor was to simply carry out the water gas process in such manner that only a small proportion of carbon monoxide was formed. Gillard found that if an excess of steam was used the carbon monoxide was oxidized to carbon dioxide, which latter can readily be eliminated from the mixture. The reaction is simply

$$
\mathrm{C}+2 \mathrm{H}_{2} \mathrm{O}=\mathrm{CO}_{2}+2 \mathrm{H}_{2} \text {. }
$$

It is, however, very difficult to carry this out in practice on a large scale, and to-day the efforts in thi direction have been practically abandoned. It is now the general practice to start out from the finishe product, water gas, and either to eliminate the carbo monoxide by absorbing agents, or to introduce in it place an equal volume of hydrogen. As absorbing agent cuprous chloride has been used, but the produst obtained by its means contains only 80 per cent hydrogen. A considerably better result is obtained accord ing to Frank and Caro, by passing water gas through retorts containing moderately heated calcium carbide. This reagent absorbs not on ly the carbon monoxide but also the carbon dioxide and the nitrogen. There is thus $o^{\prime}$ tained a very pure gas, and in addition to thi two valuable by-products, namely, graphite and calcium cyanamide. A still simpler method of eliminat ing the carbon monoxide is by liquefaction. This process also has been introduced into practice by Frank and Caro in collaboration with Linde. Th liquid carbon monoxide obtained as a by-product is utilized in a gas motor which furnishes the power for compressing the water gas. Still another process ha been worked up by the Chemische Fabrik Griesheim Elektron, in which the carbon monoxide is replaced by al equal volume of hydrogen. The principle on which this process is based is as follows: When moist wate gas is passed over lime heated to $500 \mathrm{deg}$. C. calcium carbonate is formed and hydrogen is liberated. $\mathrm{CO}+\mathrm{H}_{2} \mathrm{O}+\mathrm{CaO}=\mathrm{CaCO}_{3}+\mathrm{H}_{2}$

In order that the product may contain as high a percentage of hydrogen as possible, it is essential that the water gas be nearly free from nitrogen, a condition which can be fulfilled by the use of specially de signed generators. Considering that this proces makes use of only water gas and lime for its raw materials, it ought to prove extremely cheap.

Very similar to the preparation of producer gas is the oil gas process. It consists in blowing oil instea of steam into a generator charged with red hot coke In this way it is possible to obtain directly a gas containing as much as 90 to 96 per cent of hydrogen A special advantage of this process is that the installa tion can readily be designed in transportable form, being mounted upon two railroad trucks. The inportance of this for aerial navigation in military oper- ations is very obvious, although it may be necessary to carry the portability even farther than is possible with this arrangement. As a matter of fact, in the Boer War the English Army carried hydrogen in steel cylinders, using camels as beasts of burden. Each pacity. For the service of the English balloons having a capacity of 1,400 cubic feet a train of fifty camels was therefore required. In the Russo-Japanese War the method employed by the Russian Army consisted in the generation of hydrogen from aluminium and caustic soda. This reaction proceeds very rapidly, rendering special cooling precautions necessary. The method is of course very expensive. Another process which is specially adapted for military purposes is based on the reaction

$\mathrm{H}_{2} \mathrm{O}+\mathrm{Si}+2 \mathrm{NaOH}=\mathrm{Na}_{2} \mathrm{SiO}_{3}+2 \mathrm{H}$.

The special advantage of this is that only two kilograms of silicon are required for the production of one cubic meter of hydrogen. This process was used by Spain in the Morocco campaign. Lastly may be mentioned the production of hydrogen from calcium hydride, a gray substance prepared by passing hydrog $\in$ n over molten calcium. This substance reacts with water, liberating hydrogen according to the equation

$$
\mathrm{CaH}_{2}+\mathrm{H}_{2} \mathrm{O}=\mathrm{Ca}(\mathrm{OH})_{2}+2 \mathrm{H}_{2} .
$$

The production of one cubic meter of hydrogen requires the use of only one kilogram of calcium hydride. The process has hithertó been used only for filling small pilot balloons, but it would seem to be well calculated to fufill the requirements of military practice.

This concludes a review of the most important processes which have been proposed, and carried out with some measure of success, for the production of balloon gas. It is impossible at the present time to judge which of the numerous methods proposed will ultimately prove the best. But this can be said, that the problem as a whole is well on the way towards solution, and that the keen competition which is at work among the several processes now recognized as practically feasible, will bring results in the near future, so that we shall soon have at our command one or more thoroughly practical processes, satisfying the various conditions imposed by the conditions of consumption.

\section{The Effect of Radium on the Higher Animals}

\section{Prof. London's Researches}

IN 1903 Danysz observed that tubes containing radium, introduced under the skin in the region of the brain or spinal cord, produced symptoms of paralysis in three hours and tetanic convulsions in six hours. The effect was greatest in the youngest an: mals. Similar results were obtained by a different method by Prof. London, the celebrated Russian physiologist, from advance sheets of whose for thcom ing work on "Radium in Biology and Medicine," as reprinted in Die Umschau, the following account is derived. Prof. London exposed mice to the radiation of 30 milligrams of radium placed above their cage, within an inch or less of the animals. On th third day of the exposure the mice became dull and sleepy and their ears became red. On the fourth day these symptoms were more serious; the hind legs became paralyzed, and the animals died on the fourth or fifth day.

In 1904 London exposed three rabbits to the action of radium in a similar manner. During two week no morbid symptoms appeared. On the sixteenth day the ears reddened and red spots appeared on other parts of the body. These spots developed into ulcers which gradually healed. Six or eight weeks after the beginning of the experiment the ears were almost entirely bald and the hair began to fall from the back. The bald places then became inflamed and ulcerated. After sixteen months the ears were greatly thickened and deformed and covered with crusts, and the en tire back was destitute of hair. In the eighth month motor disturbances appeared. The animals gradually lost control of their hind legs, until finally they moved forward by means of their fore legs alone, drag ging themselves on their bellies with their hind legs trailing helplessly.

The eyes were frequently examined and in all cases were found more or less affected. The greatest changes were produced in the retina. As death approached the eyes were generally quite closed and covered with a thick secretion. The retina was not equally affected in all cases, probably because the radium rays fell upon it only when the eye was $d i$ rected toward the tube.

For several months no effect on the sexual functions was observed and the female gave birth to three litters, in July, September and November. Afterwards the sexual impulse progressively weakened and finally vanished.

Despite the fact that the injurious influence of the radiation soon appeared, the rabbits steadily in creased in weight at

London also investigated the influence of radium
Lond rapidly. emanation on frogs and white mice. Two frogs wer placed in two 2-quart glass bottles containing a lit the water. One of these bottles was connected for tw evolved, and was then disconnected and corked. After three days it was uncorked and allowed to re main open 48 hours, and was then again connected with the radium vessel for 48 hours, after which it was again disconnected and corked. When the ex periment was arranged in this manner, the frog died three or four days after the second dose of emanamained in perfect health.

The same result can be obtained by permanently connecting one of the bottles with the source of ema nation. In this method the successive phases of th effect can be followed more satisfactorily. The mor bid symptoms, which begin to appear on the sixth or seventh day, include sluggishness of movement, sleepiness, slimy appearance of the skin and difficulty in respiration, which causes death on the thirteenth to fifteenth day. The attitude of the frog and the appearance of the water are characteristic. In the con trol vessel, the frog stands in a normal manner on all fours and the water remains clear, but in the vessel exposed to emanation the frog's head and

extended forward and the water is turbid.

The frog exposed to the emanation exhibits a radio-
activity which can be detected on the first day. Very interesting experiments can be made in the dark with frogs killed in this way. Thus it can be shown that the frog emits Alpha, Beta and Gamma rays, and photographs itself on a sensitive plate covered with black paper. The imprint is most distinct when the exposure is continued three hours. The most strong ly radio-active portion is the skin. If part of the skin is removed and the experiment is repeated, the outline of the section removed appears distinctly in the photograph. The blood was found very dark and the skin abnormally soft. The microscope showed extensive changes in the skin and spinal cord.

In another experiment three or four suckling mice were placed in a bottle into which radium emanation had been admitted during 48 hours. The bottle was then corked and the mice were left ex- osed to the action of the emanation for four hours, after which the bottle was uncorked, the mice remaining in it two hours longer. The same number of mice of the same litter were placed in another bottle, not containing emanation, for comparison. Dur. ing the entire experiment and the two following days the mice showed no abnormal symptoms. On the third day the mice which had been exposed to the emanations lay on their sides, breathed with difficulty, and died with symptoms of dyspnoea. Death came more quickly in proportion to the length of exposure to emanation. The cause of death is probably to be sought in a disturbance of respiration.

Electricity from Falling Drops of Water

Søme time ago, A Schmauss showed that drops of water falling through ionized air take up a negative charge. Seeliger experimenting with ordinary water in Munich, found that in ordinary air no negative charge was gained, and when ionized by Röntgen rays only a quite feeble negative charge was assumed. To explain this, Seeliger supposes a charge effect due to Volta p.d. The author in the present paper seeks to show that Seeliger's explanation is not tenable, and, further, goes on to explain the cause of the want of greement between his own results and those of Seeliger. It appears that the taking up on an ionic charge from the air depends on the kind of water used, and while Schmauss's result is obtained with distilled water, Munich tap water behaves in the way described by Seeliger. Experiments with $\mathrm{NaCl}$ solution show that this assumes a positive charge when it falls in drops through ionized air, and, in general, Seeliger's results may be explained by the following experimental facts: (1) Distilled water which ex hibits a positive charge as Lenardeffect, takes up a negative charge in the drop experiments; (2) $\mathrm{NaC}$ solutions (and perhaps also Munich tap water) which shows a negative charge as Lenard-effect, takes a positive charge. The opposite behavior of distilled water and $\mathrm{NaCl}$ solution shows that a charge action caused through Volta p.d. of the metallic parts of the apparatus is not the explanation of the effects, but that there must rather be assumed specific forces acting between the liquid drops and the surrounding positive and negative ions. The drops may draw to themselves those ions whose charge is opposite to their own on account of the charge arising from the Lenard double layer.-Annalen der Physik. 